\title{
Regularized super-resolution restoration algorithm for single medical image based on fuzzy similarity fusion
}

\author{
Xingying $\mathrm{Li}^{1}$ and Weina $\mathrm{Fu}^{2,3^{*}}$ (D)
}

\begin{abstract}
Medical images are blurred and noised due to various reasons in the acquirement, transmission and storage. In order to improve the restoration quality of medical images, a regular super-resolution restoration algorithm based on fuzzy similarity fusion is proposed. Based on maintained similarity in multiple scales, the fused similarity of the medical images is computed by fuzzy similarity fusion. First, fuzzy similarity is determined by the regional features. The images with certain similarity are obtained according to the maximum value, and the fused image is obtained by all obvious regional features. Then, an adaptive regularized restoration algorithm is employed. In order to ensure the objective function has a global optimal solution, regularized parameters of the global minimum solution of nonlinear function are solved iteratively. Finally, experimental results show that mean square error (MSE) and peak signal-to-noise ratio (PSNR) of the restored image are visibly improved. The restored image also has an obvious improvement in the burr of local edge. Moreover, the algorithm has good stability with significantly enhanced PSNR.
\end{abstract}

Keywords: Fuzzy similarity, Fusion, Medical image, Regular super-resolution, Image restoration, Burr of edge

\section{Introduction}

In clinical practice, imaging is widely used in medical diagnosis and treatment because of its high safety and strong real-time performance. With the rapid development of medical technology, a large number of highresolution images have emerged, such as computed tomography (CT), positron emission tomography (PET), magnetic resonance imaging (MRI), diffuse weighted imaging (DEI), single photon emission computed tomography (SPECT), etc. These imaging techniques provide a variety of functional and anatomical information at different spatial and temporal resolutions [1]. All these kinds of medical images with super-resolution play an important role in life science research, medical diagnosis and clinical treatment. Therefore, super-resolution restoration improves the resolution of medical images, realizes the detection and recognition of more subtle

\footnotetext{
* Correspondence: fwn0124@yeah.net

${ }^{2}$ College of Compute and Information Enginnering, Inner Mongolia

Agricultrual University, Hohhot 010018, China

${ }^{3}$ College of Information Science and Engineering, Hunan Normal University,

Changsha 410081, China

Full list of author information is available at the end of the article
}

lesions, and assists doctors to provide more accurate information for reducing the misdiagnosis rate.

However, original medical images are far from these requirements. Therefore, it is of great significance to recover the acquired image, which plays an important role in image recognition, detection and classification. Obtaining satisfied high-resolution images by improving physical hardware is difficult and expensive. Therefore, the restoration technology that improves the image resolution through software has become a research hotspot [2].

For restoration of single image with traditional methods, since only one input image is utilized, the enhancement of restoration and resolution is greatly limited [3]. Therefore, current research focuses on superresolution image restoration based on sequence images by assuming that imaging conditions (light, lens focal length, etc.) at different times are identical with no major change in an one-time application (such as large deformation, occlusion) [4]. However, the registration accuracy, which is the key to super-resolution recovery by using sequence images, is affected heavily because these conditions are difficult to fully satisfy. SuperResolution (SR) refers to recover a high-resolution image 
(HR) with a richer detail from low-resolution image (Lower Resolution, LR) with multi-frames, and it soon becomes a hotspot in medical imaging [5]. The SR technique is based on some prior knowledge of image degradation, which is degraded to classic image restoration when the impulse response of the system (the point spread function) is known. However, in most cases, the point spread function is difficult to determine. In this way, super-resolution image restoration extracts the degradation information from the observed image in a specific way to find out image restoration method.

Current research of the super-resolution has made some achievements in image restoration algorithms. However, its application range has certain limitations. Jeong et al. assumed that the Point Spread Function (PSF) was a circle and used a cyclic square matrix as the degenerate matrix to use fuzzy system in superresolution image estimation [6]. Their method is an iteration which identifies PSD and estimate real image iteratively. In the iteration, they set one in PSD and real image to a known result when the other is estimating by generalized crossover criteria. Meantime, Alqadah et al. effectively dealt PSF with the Linear Spatial Invariant (LSI) fuzzy conditions, and estimated the fuzzy parameters by Gaussian quadratic criterion and Lanczos algorithm [7]. They also improved the secondary and cyclical requirements of the PSF matrix, and increased robustness and effectiveness of the algorithm. Recently, an adaptive regular blind reconstruction algorithm was also proposed by a regular processing based on local smooth features of images [8].

In order to improve the resolution of medical images, it is considered to constrain the super-resolution solution space for LR images by using fusion of fuzzy similarity and multi-scale retention of the image. This paper studies the regularized super-resolution restoration of medical images to improve restoration effectiveness. Section 2 provides materials and methods used in this paper; Section 3 provides results of our method by experiments; Section 4 discussed the results and Section 5 concludes the whole paper.

\section{Materials and methods}

\subsection{Super resolution analysis}

In general, function of medical image degradation is unknown. Super-resolution image restoration assumes that the LR sequence is an LSI degenerate of the HR image [9]. Blurr ing, down-sampling, and displacement of image are simulated separately. Then, because downsampling and blurring are unchanged during the formation of the LR sequence, the LR frame is shown in Eq. 1.

$$
y_{k}=D B M_{k}+n_{k}=H_{k} x+n_{k} \quad 1 \leq k \leq p
$$

where $x, y_{k}$ and $n_{k}$ are the vector of the restored HR medical image, the $k$-th frame of degraded image and its additive noise, respectively, the matrices $D, B$ and $M_{k}$ are the down-sampling operator of the imaging system, the fuzzy operator, and the displacement operator of the $k$ frame medical image, respectively, $H_{k}$ is the degradation matrix of the $k$-th frame of medical image. In Eq. $1, D$ is determined by the down-sampling rate, $M_{k}$ is estimated by the inter-frame displacement, and the fuzzy operator $B$ is related to the PSF. $B$ and $n_{k}$ are generally not directly available and need to be estimated from the LR sequence.

A general super-resolution algorithm adds detail of medical image and improves resolution by fusing all the information in the LR sequence [10]. Assuming that the size of the restored LR medical image is $M \times N$, and the resolution of the medical image is enhanced by $r$ times. The Eq. 2 is provided by adding Eq. 1 when $\mathrm{k}$ is set from 1 to $\mathrm{p}$.

$$
y=H x+n
$$

where $y \in R^{p M N \times 1}, \quad H \in R^{p M N \times r^{2} M N}, \quad n \in R^{p M N \times 1}, \quad x \in$ $R^{r^{2} M N \times 1}$. Therefore, a high-dimensional calculation is required to recover HR medical images from Eq. 2 even for a medium-scale LR image sequence.

Since the fuzzy operator $B$ is a discrete matrix form of PSF with finitely support, the matrix $B$ is degraded [11]. Furthermore, in order to fully recover a true HR medical image, an image sequence containing $r^{2}$ frames that includes all non-redundant complementary information is needed. However, the frames' number of LR image in practical applications is often limited, which makes Eq. 2 an ill-conditioned equation. Therefore, regularization is used to solve the problem in this paper.

It is assumed that SR has been accurately estimated in medical image motion, and the blur of image is unknown. In order to simplify the estimation of image blur, we assume that the structure of the PSF is known and the parameters of PSF are unknown. The fuzzy matrix $B$ is re-expressed as a function with parameter $\sigma$ by Eq. 3 .

$$
y=H(\sigma) x+n
$$

In this way, restoration of SR medical image estimates the HR medical image from LR images with $p$ frames by Eq. 3.

\subsection{Fuzzy similarity fusion strategy based on local feature information}

\subsubsection{Fuzzy similarity}

If the pixel matrix of a fused image is regarded as a twodimensional fuzzy set, according to the fuzzy mathematical theory, similarity degree between the two fuzzy sets is measured by the closeness of them $[12,13]$. In a general data fusion process, it is assumed that there are $N$ sensors to measure the same feature indicator in the 
same information collection system respectively. Let $x_{i}$ denotes the data measured by the $i$-th sensor at a certain time and the random variable $X_{i}$ denotes an observation corresponding to $x_{i}$. Due to the randomness of external disturbance factors, the true degree of $X_{i}$ is only determined by the information which is hidden in the data $x_{1}$, $x_{2}, \cdots x_{N}$. The authenticity of $x_{i}$ is high if the similarity between $x_{i}$ and the rest data is high [14].

Then, similarity function in fuzzy mathematics is used to quantify the similarity of the observations in each sensor at the same time [15]. Though there are several ways to define the similarity function, the following two definitions are selected by applying low computational complexity. At time $k$, the similarity of the data measured by $N$ sensors is:

$$
\alpha_{1,2, \cdots, N}(k)=\frac{\min \left\{x_{1}(k), x_{2}(k), \cdots, x_{N}(k)\right\}}{\max \left\{x_{1}(k), x_{2}(k), \cdots, x_{N}(k)\right\}}
$$

or

$$
\alpha_{1,2, \cdots, N}(k)=\frac{\mathrm{N} \min \left\{x_{1}(k), x_{2}(k), \cdots, x_{N}(k)\right\}}{x_{1}(k), x_{2}(k), \cdots, x_{N}(k)}
$$

where $x_{i}(k)$ is the result of the $k$-th sampling of the $i$-th sensor, and $\alpha_{1,2, \cdots, N} \in(0,1]$. When the value is 1 , it means that the $k$-th sampling result of $N$ sensors is the same, and the maximum similarity is obtained at this time. When the value is close to 0 , it means that the $k$-th sampling result of $N$ sensors is almost different and has the smallest similarity.

\subsubsection{Regional features}

In order to directly extract or weight the average extracted pixels from the fused medical image, Eqs. 4-5 are used to describe the relation degree of the regional features around each pixel. Since the source image is obtained through a certain pre-processing algorithm, it contains a few noise and has a good visual effect. Therefore, the key point of improvement for the fusion effect is the abundant details of the fused image. In this way, the regional variance, regional gradient, and regional mean are used as regional features [16]. The medical image contains more details and the fusion effect of medical image is better when these indicators are large enough.

If a pixel point $I(i, j)$ is centered, the area variance, region gradient, and region mean of point $I(i, j)$ are defined as:

$$
\begin{aligned}
& V(i, j)=\frac{1}{d} \sum_{m=i-k}^{i+k} \sum_{n=j-k}^{j+k}[I(m, n)-\bar{I}(m, n)]^{2} \\
& G(i, j)=\frac{1}{d} \sum_{m=i-k}^{i+k} \sum_{n=j-k}^{j+k}\left[G_{x}^{2}(m, n)-G_{y}^{2}(m, n)\right]^{\frac{1}{2}} \\
& M(i, j)=\frac{1}{d} \sum_{m=i-k}^{i+k} \sum_{n=j-k}^{j+k}[I(m, n)-\bar{I}(m, n)]
\end{aligned}
$$

where $d$ is the number of pixels in the area with size $(2 k+1) \times(2 k+1)$ centered on the point $I(i, j), G^{x}(m, n)$ and $G^{y}(m, n)$ represent the first order derivative of the medical image in horizontal direction and vertical direction at the point $(m, n)$, respectively. Then, $\bar{I}(m, n)$ is used to represent the average of the pixels in the area by Eq. 9.

$$
\bar{I}(m, n)=\frac{1}{d} \sum_{m=i-k}^{i+k} \sum_{n=j-k}^{j+k} I(m, n)
$$

Therefore, the regional feature $c(i, j)$ is used as the measurement data of sensor [17]. Similarity $\alpha_{1,2}, \cdots, N$ of this pixel point in the plurality of fused images is obtained by using Eq. 4 or Eq. 5. Thereby, we select a corresponding extraction to obtain an approximate value of the pixel point. In real application, when the similarity $\alpha_{1,2}, \cdots, N$ is lower than a defined lower threshold $T_{\alpha}\left(T_{\alpha}>0\right)$, the regional features $c_{1}(i, j), c_{2}(i, j), \cdots c_{N}(i, j)$ are considered to be completely different. A general value of $T_{\alpha}$ is between 0.3 to 0.5 .

\subsubsection{Rules of fusion decision}

Determining the decision rules is the core of fusion strategy. It refers to how we can select information from the source image and add it into the fused image [18]. The simplest method is that we take the mean of source image coefficients as the coefficients of fused image. However, this fusion strategy reduces the contrast of results. It is known from the optical system imaging principle that the high frequency component of sharp image is much larger than blurred image [19]. If gradient, variance, and mean value of a certain area are larger, the more high-frequency information is included in the area and image information is richer. Therefore, the choice of pixels is guided by the size of region gradient, variance, and mean. This decision rule is called the "maximum selection" [20]. The "maximum selection" rule will reach the best result if only one image provides the most useful information in the corresponding position of source image. However, not all fusion source images satisfy this assumption in reality. Therefore, the following decision rules are used:

$$
\begin{aligned}
I(i, j) & =\left\{\begin{array}{c}
I_{k}, \arg \max \left\{c_{1}(i, j), c_{2}(i, j), \cdots c_{N}(i, j)\right\} \\
\alpha_{1,2, \cdots, N} \leq T_{\alpha} \\
\omega_{1}(i, j) I_{1}(i, j)+\omega_{2}(i, j) I_{2}(i, j)+\cdots+ \\
\omega_{N}(i, j) I_{N}(i, j), \alpha_{1,2, \cdots, N}>T_{\alpha}
\end{array}\right. \\
\omega_{k}(i, j) & =\frac{c_{k}(i, j)}{c_{1}(i, j)+c_{2}(i, j)+\cdots+c_{N}(i, j)} \\
& =1,2, \cdots, \mathrm{N}
\end{aligned}
$$

where $T_{\alpha}$ is the threshold and $I$ is the fused image. The 
similarity $\alpha_{1,2}, \cdots, N$ plays a decision-making role in the integration. When the similarity is small, it is considered that only one image provides useful information. At this time, the extraction strategy of fused image is "take one from multiple", that is, the pixel with best fusion quality is extracted as the final pixel point of the fused image according to region features, and other pixels at same position are dropped. When the similarity is large, the extraction strategy of medical image is "multiple fusion", that is, the pixel points of the plurality of medical images are weighted and averaged based on regional features to obtain the pixels of the fused image.

The innovation of this decision rule is as follows.

A weighted combination of these medical images is used as the fused image, and the weight coefficient is determined by regional features at the pixel for a plurality of medical images with a certain degree of relevance [21, $22]$. It reduces noise and ensures the stability of fusion when the source image contains similar information. The saliency information in the source image is preserved when no similar information is included.

Through the above process, multiple medical images are fused based on the fuzzy similarity fusion to obtain a single medical fusion image. Then, a single medical image is restored by using a regularized super-resolution restoration algorithm.

\subsection{Adaptive regularization super-resolution restoration}

Super-resolution image restoration is essentially a morbid problem that needs to be solved by a regularization algorithm:

$$
L(x)=\sum_{k=1}^{K}\left(y_{k}-H_{k} x\right)^{T}\left(y_{k}-H_{k} x\right)+\alpha\|C x\|^{2}
$$

Considering the penalty effect of similarity in value function, we have

$$
\begin{aligned}
L(x) & =\sum_{k=1}^{K} L_{k}(x) \\
& =\sum_{k=1}^{K}\left[\left(y_{k}-H_{k} x\right)^{T} S_{k}\left(y_{k}-H_{k} x\right)+\alpha_{k}(x)\|C x\|^{2}\right]
\end{aligned}
$$

where $\alpha_{k}(x)$ is a regular parameter, $C$ is a smoothing operator, and is generally taken as a Plass operator.

\subsubsection{Selection of regular parameters}

A regular parameter is selected to make the nonlinear function $L(x)$ have a global minimum solution. In fact, $L(x)$ has a global minimum solution if $L(x)$ is guaranteed to be a convex function [23, 24]. Also if $L_{k}(x)$ is a convex function, $L(x)$ is a convex function by property of the convex function. Therefore, in order to make that the general function $L(x)$ has a global minimum solution, it is only necessary to ensure that $L_{k}(x)$ is a convex function.

Proposition 1. If regular parameters satisfy the following two attributes, $\mathrm{L}_{\mathrm{k}}(\mathrm{x})$ is a convex function.

(1) $\alpha_{k}(x)$ is a linear, monotonically increasing function of $L_{k}(x)$ :

$$
\alpha_{k}(x)=f\left(L_{k}(x)\right)+\gamma_{k} L_{k}(x)
$$

that is

$$
\alpha_{k}(x)=\frac{\left(y_{k}-H_{k} x\right)^{T} S_{k}\left(y_{k}-H_{k} x\right)}{\frac{1}{\gamma_{k}}-\|C x\|^{2}}
$$

(2)

$$
\frac{\partial f\left(L_{k}\right)}{\partial L_{k}}<\frac{1}{\|C x\|^{2}}
$$

Proof. Let

$$
L_{k}(x)=L_{k}(p, q)=p+f\left(L_{k}(p, q)\right) q
$$

where $p=\left(y_{k}-H_{k} x\right)^{T} S_{k}\left(y_{k}-H_{k} x\right), q=\|C x\|^{2}$.

To make $L_{k}(x)$ a convex function, $\forall z_{1}, z_{2}$ and $0<\lambda<1$ we have

$$
\begin{aligned}
L_{k}(x)= & \left(\lambda z_{1}+(1-\lambda) z_{2}\right) \leq \lambda L_{k}\left(z_{1}\right) \\
& +(1-\lambda) L_{k}\left(z_{2}\right)
\end{aligned}
$$

where $z_{1}=\left(p_{1}, q_{1}\right)^{T}, z_{2}=\left(p_{2}, q_{2}\right)^{T}$, that is

$$
\begin{aligned}
L_{k}= & \left(\lambda p_{1}+(1-\lambda) p_{2}, \lambda q_{1}+(1-\lambda) q_{2}\right) \\
& \leq \lambda L_{k}\left(p_{1}, q_{1}\right)+(1-\lambda) L_{k}\left(p_{2}, q_{2}\right)
\end{aligned}
$$

According to Eq. 17, Eq. 19 is converted into:

$$
\begin{aligned}
\lambda q_{1} f & \left(L_{k}\left(\lambda p_{1}+(1-\lambda) p_{2}, \lambda q_{1}+(1-\lambda) q_{2}\right)\right)+ \\
& (1-\lambda) q_{2} f\left(L_{k}\left(\lambda p_{1}+(1-\lambda) p_{2}, \lambda q_{1}+(1-\lambda) q_{2}\right)\right) \\
& \leq \lambda q_{1} f\left(L_{k}\left(p_{1}, q_{1}\right)\right)+(1-\lambda) q_{2} f\left(L_{k}\left(p_{2}, q_{2}\right)\right)
\end{aligned}
$$

Since $\alpha_{k}(x)$ satisfies Eq. 14, Eq. 20 is further transformed into:

$$
\begin{aligned}
& \lambda q_{1} L_{k}\left(\lambda p_{1}+(1-\lambda) p_{2}, \lambda q_{1}+(1-\lambda) q_{2}\right)+ \\
& \quad(1-\lambda) q_{2} L_{k}\left(\lambda p_{1}+(1-\lambda) p_{2}, \lambda q_{1}+(1-\lambda) q_{2}\right) \\
& \quad \leq \lambda q_{1} L_{k}\left(p_{1}, q_{1}\right)+(1-\lambda) L_{k}\left(p_{2}, q_{2}\right)
\end{aligned}
$$

If $L_{k}(x)$ is a convex function, then 


$$
\begin{aligned}
& \lambda q_{1} L_{k}\left(\lambda p_{1}+(1-\lambda) p_{2}, \lambda q_{1}+(1-\lambda) q_{2}\right)+ \\
& (1-\lambda) q_{2} L_{k}\left(\lambda p_{1}+(1-\lambda) p_{2}, \lambda q_{1}+(1-\lambda) q_{2}\right) \leq \\
& \lambda q_{1}\left[\lambda L_{k}\left(p_{1}, q_{1}\right)+(1-\lambda) L_{k}\left(p_{2}, q_{2}\right)\right]+ \\
& (1-\lambda) q_{2}\left[\lambda L_{k}\left(p_{1}, q_{1}\right)+(1-\lambda) L_{k}\left(p_{2}, q_{2}\right)\right]= \\
& \lambda^{2} q_{1} L_{k}\left(p_{1}, q_{1}\right)+(1-\lambda)^{2} q_{2} L_{k}\left(p_{2}, q_{2}\right)+\lambda(1-\lambda) \\
& {\left[q_{1} L_{k}\left(p_{2}, q_{2}\right)+q_{2} L_{k}\left(p_{1}, q_{1}\right)\right]}
\end{aligned}
$$

Eq. 21 is equivalent to following Eq. 23.

$$
\begin{aligned}
& \lambda q_{1} L_{k}\left(p_{1}, q_{1}\right)+(1-\lambda) q_{2} L_{k}\left(p_{2}, q_{2}\right)- \\
& \lambda^{2} q_{1} L_{k}\left(p_{1}, q_{1}\right)-(1-\lambda){ }_{2} q_{2} L_{k}\left(p_{2}, q_{2}\right)- \\
& \lambda(1-\lambda)\left[q_{1} L_{k}\left(p_{2}, q_{2}\right)+q_{2} L_{k}\left(p_{1}, q_{1}\right)\right]= \\
& \lambda(1-\lambda)\left(q_{1}-q_{2}\right)\left[L_{k}\left(p_{1}, q_{1}\right)-L_{k}\left(p_{2}, q_{2}\right)\right]
\end{aligned}
$$

Because $\lambda(1-\lambda)>0$, Eq. 23 is equivalent to Eq. 24 .

$$
\left(q_{1}-q_{2}\right)\left[L_{k}\left(p_{1}, q_{1}\right)-L_{k}\left(p_{2}, q_{2}\right)\right] \geq 0
$$

We have Eq. 25 because $L_{k}(p, q)$ is a monotonically increasing function for $\mathrm{q}$ by Eq. 24 [25].

$$
\frac{\partial L_{k}}{\partial q} \geq 0
$$

Because $\quad L_{k}=p+f\left(L_{k}\right) q, \frac{\partial L_{k}}{\partial q}=\frac{\partial f}{\partial L_{k}} \cdot \frac{\partial L_{k}}{\partial q} \bullet q+f\left(L_{k}\right)$, which is same as

$$
\frac{\partial L_{k}}{\partial q}=\frac{f\left(L_{k}\right)}{1-q \frac{\partial f}{\partial L_{k}}} \geq 0
$$

And because of $f\left(L_{k}\right) \geq 0$, thus

$$
\frac{\partial f}{\partial L_{k}}<\frac{1}{q}=\frac{1}{\|C x\|^{2}}
$$

Proposition 1 is proved.

\subsubsection{Iterative solution}

The selection of regularized parameters makes $L(x)$ a convex function. Thus, $L(x)$ has a global minimum solution $\hat{x}$, then $\Delta_{x} L(\hat{x})=0$, and $\Delta_{x} \sum_{k=1}^{K} \alpha_{k}(\hat{x})=0$.

Because

$$
\begin{aligned}
\Delta_{x} L_{k}(x)= & -2 H_{k}^{T} S_{k}\left(y_{k}-H_{k} x\right)+\|C x\|^{2} \Delta_{x} \alpha_{k}(x) \\
& +2 \alpha_{k}(x) C^{T} C x
\end{aligned}
$$

Then,

$$
\begin{aligned}
\Delta_{x} L_{k}(x)= & 2 \sum_{k=1}^{K}\left\{\left(H_{k}^{T} S_{k} H_{k}+\alpha_{k}(x) C^{T} C\right) x-H_{k}^{T} S_{k} y_{k}\right\} \\
& +\sum_{k=1}^{K}\|C x\|^{2} \Delta_{x} \alpha_{k}(x)
\end{aligned}
$$

Thus, an iterative formula of $\mathrm{x}$ is obtained by Eq. 30 .

$$
x_{n+1}=x_{n}-\varepsilon \sum_{k=1}^{K}\left\{\left(H_{k}^{T} S_{k} H_{k}+\alpha_{k}(x) C^{T} C\right) x_{n}-H_{k}^{T} S_{k} y_{k}\right\}
$$

\section{Results}

With experimental comparisons between the two different provided similarities, we find the improved signal-tonoise ratio (ISNR) of the fused image is similar, and experimental results are almost the same. In this way, this paper chooses Eq. 4 as the similarity measure.

\subsection{Recovery analysis of medical images}

A medical image with size $256 \times 256$ pixels is used as the test image in our experiment. The test image is convolved by using the point spread function of a $5 \times 5$ Gaussian matrix. Then, horizontal and vertical directions are down-sampled to produce an LR image of $128 \times 128$ pixels by the factor 2 , which is used as a reference frame. Moreover, 5 LR image frames with size $128 \times 128$ are generated with fuzzy similarity matching, and a total of 6 frames of LR images are obtained. Finally, numerical experiment is carried out by using the proposed algorithm, and image restoration result is compared with Ref. [3] and Ref. [5]. The results are shown in Fig. 1 and Table 1.

Evaluation of the results is generally divided into two types. One is subjective evaluation, considering the human eye's feelings for the details of restored image. The other is objective evaluation criterion, generally using MSE and PSNR. Assuming $x$ is the original image, $\hat{x}$ is the restored image, and $N$ is the number of pixels, then MSE and PSNR are shown in Eqs. 31-32.

$$
\begin{aligned}
M S E & =\frac{1}{N} \sum_{i=0}^{N-1}\left(x_{i}-\hat{x}_{i}\right)^{2} \\
P S N R & =10 \lg \frac{255^{2}}{\frac{1}{N} \sum_{i=0}^{N-1}\left(x_{i}-\hat{x}_{i}\right)^{2}}
\end{aligned}
$$

Figure 1 shows medical image restoration effects of different algorithms. Recovery effects of the three algorithms are compared in Table 1. Compared with Liang's algorithm, mean square error and peak signal-to-noise ratio of the reconstructed image are significantly improved. Compared with Wu's algorithm, peak signal-tonoise ratio and mean square error of the restored image are slightly improved a little. However, from the restoration of visual effects, it is significantly better than Wu's algorithm. It is mainly manifested that the image restored by the proposed algorithm has a significant 


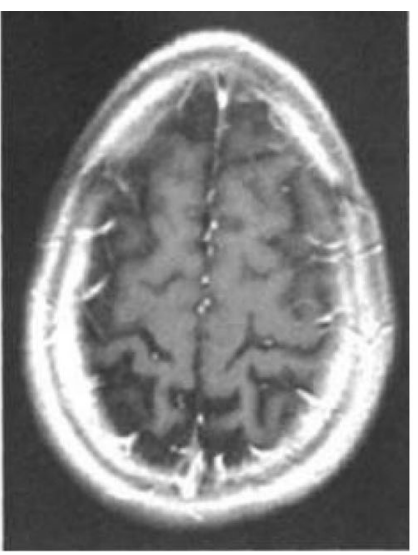

(a) Primitive medical images (256 x 256)

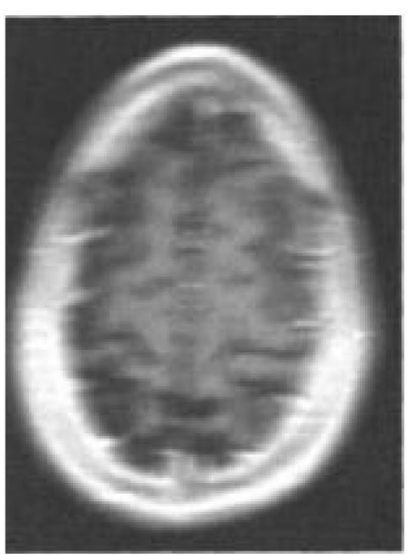

(b) LR image with Gauss blur

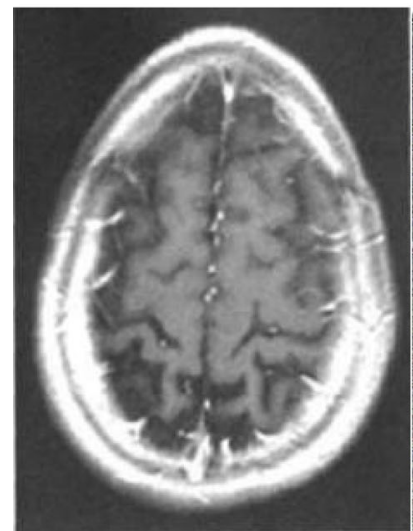

(c) Proposed algorithm

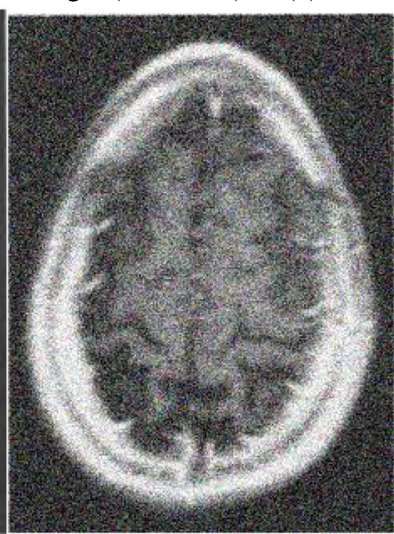

(d) Liang's algorithm [3]

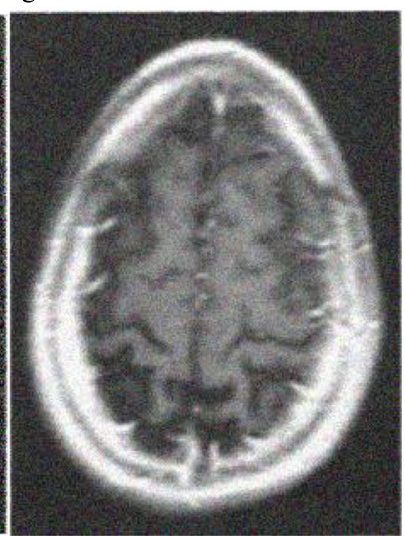

(e) Wu's algorithm [5]

Fig. 1 Comparison of restoration results of different restoration methods. a Primitive medical images $(256 \times 256)$. b LR image with Gauss blur. c Algorithm in this paper. d Liang's algorithm. e Wu's algorithm

improvement on the local edge glitch phenomenon, which is due to the proposed fuzzy similarity fusion algorithm.

\subsection{Fusion analysis of fuzzy similarity}

Standard medical image $1(512 \times 512)$ and image 2 $(512 \times 512)$ are selected for testing. First, gray value of the real image is normalized. The image is then subjected to fuzzy convolution and noise addition to obtain a contaminated image. The PSF of the fuzzy convolution uses a Gaussian function with a standard deviation of 10. Additive noise is Gaussian white noise with a variance of 0.001 . Quality of the fused image is measured in terms of ISNR.

Table 1 Comparison of the effects of different methods of single hospital

\begin{tabular}{lll}
\hline & MSE & PSNR \\
\hline Paper algorithm & 237.11 & 24.38 \\
Liang's algorithm [3] & 141.91 & 26.61 \\
Wu's algorithm [5] & 139.02 & 26.7 \\
\hline
\end{tabular}

Experiment 1: the image processed by different wavelet bases are used as fused source images, and then image fusion is performed by the proposed algorithm. Comparing the performance with fusion image, the results are shown in Tables 2 and 3.

It is known that fusion image's ISNR of the proposed algorithm is significantly improved from Tables 2 and 3 . For example, in the fourth group of experiments for image 2 in Table 3, source image is an image restored by wavelets $\mathrm{db} 3$, sym8, sym10, and ISNR thereof is 7.49, 7.53 and 7.54, respectively. ISNR of the restored image after restoration is $8.01,8.01$ and 8.06 , which is $0.5 \mathrm{~dB}$ higher than ISNR before fusion, and fusion effect is obvious.

Experiment 2: images from different Contourlet bases and images from different wavelet bases are used as fusion source images to compare the image performance using a single Contourlet basis (Table 4) and the performance with fusion image (Table 5).

It is known that ISNR of the image after fusion is significantly improved from Tables 4 and 5. For example, in the first group of experiments in Table 5, for image 1, ISNR of the image after (9/7, 9/7), (sym8, 9/7), sym8, 
Table 2 Performance analysis of wavelet-based image fusion algorithms

\begin{tabular}{|c|c|c|c|c|c|}
\hline \multirow{2}{*}{$\begin{array}{l}\text { Wave } \\
\text { filter }\end{array}$} & \multicolumn{2}{|l|}{ Image } & \multirow{2}{*}{$\begin{array}{l}\text { Wave } \\
\text { filter }\end{array}$} & \multicolumn{2}{|l|}{ Image } \\
\hline & Medical image 1 & Medical image 2 & & Medical image 1 & Medical image 2 \\
\hline db1 & 4.11 & 6.95 & coif1 & 4.39 & 7.35 \\
\hline db2 & 4.38 & 74 & coif2 & 4.61 & 7.52 \\
\hline db3 & 4.51 & 7.49 & coif3 & 1.69 & 7.57 \\
\hline $\mathrm{db} 4$ & 4.58 & 4.46 & coif5 & 4.74 & 7.55 \\
\hline db5 & 4.64 & 7.46 & bior1.1 & 4.11 & 6.95 \\
\hline db6 & 4.67 & 7.42 & bior1.3 & 4.1 & 6.94 \\
\hline db7 & 4.68 & 7.43 & bior2.2 & 3.74 & 6.89 \\
\hline $\mathrm{db} 8$ & 4.68 & 7.4 & bior2.6 & 3.75 & 6.88 \\
\hline db15 & 4.69 & 7.23 & bior3.1 & 3.17 & 6.07 \\
\hline sym2 & 4.38 & 7.34 & bior3.5 & 3.07 & 6.09 \\
\hline sym3 & 4.51 & 7.9 & bior4.4 & 2.45 & 5.47 \\
\hline sym8 & 4.72 & 7.53 & bior6.8 & 4.6 & 6.78 \\
\hline sym10 & 4.74 & 7.54 & & & \\
\hline
\end{tabular}

and coif5 fusion is $4.29,435,4.72$, and 4 . 74 , respectively. ISNR of the fused image processed by the proposed algorithm is 4.85 and 4.82, which is improved by $0.1-0.6 \mathrm{~dB}$, and the effect is improved obviously. Combined with the results of the first set of experiments, the proposed algorithm has a good fusion effect.

\subsection{Stability analysis of the algorithm}

The experiment tests stability of the algorithm. For medical images of different sizes, image restoration is performed using three different restoration algorithms. The image restoration results of different algorithms are compared in Table 6.

By analyzing Table 6, when medical image size is 1 $\mathrm{MB}$, difference between MSE and PSNR of the three algorithms is both small. The obtained MSE and PSNR values by the proposed algorithm show a gradual ascending and downward trend with continuous increment of medical image size when medical images with different sizes are restored. However, the results obtained by Ref. [3] and Ref. [5] show an upward trend and a downward trend with more significant volatility when the size of medical image increases. In this way, experimental results show that stability of the proposed algorithm is better than traditional algorithms.

\subsection{Comparative analysis of image restoration integrity}

Taking the integrity of image restoration as an index, the proposed algorithm in this paper is compared with Ref. [3] and Ref. [5]. The results are shown in Fig. 2:

From Fig. 2, we find that the algorithm of this paper has the highest recovery integrity, especially when the pixel is 300, the recovery of this algorithm reaches a maximum of $90 \%$ for medical images with different pixel conditions. Liang's algorithm is the algorithm with the lowest complete degree of image restoration in the three algorithms. The maximum degree of integrity is $41 \%$, and the image restoration integrity of the algorithm of

Table 3 Performance analysis of the algorithm in this paper

\begin{tabular}{|c|c|c|c|c|c|c|c|}
\hline \multirow{2}{*}{$\begin{array}{l}\text { Experimental } \\
\text { serial number }\end{array}$} & \multirow[t]{2}{*}{ Filter combination } & \multicolumn{3}{|c|}{ Medical image 1} & \multicolumn{3}{|c|}{ Medical image 2} \\
\hline & & Gradient & Variance & Mean value & Gradient & Variance & Mean value \\
\hline 1 & $\mathrm{db} 1+$ sym8 + sym10 & 4.8 & 4.83 & 4.82 & 7.84 & 7.85 & 7.91 \\
\hline 2 & sym8 + sym10 + coif5 & 4.84 & 4.84 & 4.84 & 7.76 & 7.76 & 7.78 \\
\hline 3 & $\mathrm{db} 1+$ sym8 + coif5 & 4.81 & 4.84 & 4.83 & 37.83 & 7.84 & 7.9 \\
\hline 4 & $\mathrm{db} 3+$ sym8 + sym10 & 4.91 & 4.93 & 4.92 & 8.01 & 8.01 & 8.06 \\
\hline 5 & $\mathrm{db} 4+$ sym8 + sym 10 & 4.91 & 4.92 & 4.91 & 7.96 & 7.96 & 8.01 \\
\hline 6 & $\mathrm{db} 1+$ sym8 & 4.67 & 4.71 & 4.7 & 7.64 & 7.67 & 7.73 \\
\hline 7 & sym8 + sym10 & 4.83 & 4.83 & 4.83 & 7.76 & 7.76 & 7.78 \\
\hline 8 & $\mathrm{db} 3+$ sym10 & 4.84 & 4.87 & 4.83 & 7.95 & 7.95 & 8.04 \\
\hline 9 & $\mathrm{db} 4+\operatorname{sym} 10$ & 4.86 & 4.88 & 4.88 & 7.92 & 7.93 & 7.98 \\
\hline
\end{tabular}


Table 4 Performance comparison of ontourlet-based restoration algorithms

\begin{tabular}{lll}
\hline (LP/DFB) & Medical image 1 & Medical image 2 \\
\hline (9/7,9/7) & 4.29 & 7.12 \\
(pkva,pkva) & 4.35 & 6.09 \\
(haar,pkva) & 4.08 & 6.59 \\
(haar,pkva6) & 3.95 & 6.19 \\
(haar,5/3) & 3.71 & 6.45 \\
(sym8,pkva6) & 3.53 & 7.14 \\
(sym8,pkva) & 4.65 & 7.3 \\
(sym8,9/7) & 4.35 & 7.13 \\
(sym8,5/3) & 3.94 & 6.83 \\
\hline
\end{tabular}

the Wu's algorithm is up to $50 \%$. It can be seen that the image restoration integrity of the proposed algorithm is high, which indicates that the regular super-resolution restoration algorithm in this paper has a strong feasibility.

\subsection{Comparative analysis of image restoration efficiency}

Taking the image restoration efficiency as an index, the algorithm in this paper is compared with Ref. [3] and Ref. [5]. The results are shown in Fig. 3.

According to Fig. 3, the overall image restoration efficiency of this algorithm is high, and the average recovery efficiency is up to $80 \%$. The restoration efficiency of algorithms in Ref. [3] and Ref. [5] first showed an upward trend, and after the pixel value is 300 , the restoration efficiency began to decrease. The average recovery efficiency of Liang's algorithm is $40 \%$, and the average recovery efficiency of Wu's algorithm is $30 \%$, which shows the superiority of this algorithm.

\subsection{Image recovery time-consuming comparison}

Taking the image recovery time-consuming as an index, the algorithm in this paper is compared with Ref. [3] and Ref. [5]. The results are shown in Table 7:

It is clearly in Table 7 that the time consuming is about $7.9 \mathrm{~s}$ when the single-amplitude medical image is recovered by the proposed algorithm, the average time consumption of the image recovery of Liang's algorithm is about $31 \mathrm{~s}$, and the average time of the image recovery

Table 5 Performance analysis of the algorithm in this paper

\begin{tabular}{lll}
\hline Filter combination & Medical image 1 & Medical image 2 \\
\hline (9/7,9/7) + (sym8,9/7) + sym8 + coif5 & 4.854 .884 .85 & 7.667 .677 .68 \\
(sym8,pkva) $+(9 / 7,9 / 7)+($ pkva,pkva) & 4.674 .684 .67 & 7.647 .647 .70 \\
$($ sym8,pkva) + sym8 + sym10 & 4.954 .974 .96 & 7.957 .947 .99 \\
(haar,5/3) + sym8 + sym10 & 4.714 .774 .70 & 7.717 .727 .79 \\
(sym8,pkva) + db4 + sym10 & 7.934 .964 .94 & 7.997 .998 .04 \\
\hline
\end{tabular}

Table 6 Comparisons of stability of different algorithms

\begin{tabular}{|c|c|c|c|c|c|c|}
\hline \multirow{2}{*}{$\begin{array}{l}\text { Image } \\
\text { size/ } \\
\text { MB }\end{array}$} & \multicolumn{2}{|c|}{ Algorithm in this paper } & \multicolumn{2}{|c|}{ Liang's algorithm } & \multicolumn{2}{|c|}{ Wu's algorithm } \\
\hline & MSE & PSNR & MSE & PSNR & MSE & PSNR \\
\hline 1 & 96.34 & 199.24 & 100.53 & 186.42 & 99.51 & 191.55 \\
\hline 10 & 96.93 & 196.57 & 112.68 & 180.66 & 106.63 & 180.02 \\
\hline 100 & 97.36 & 194.84 & 139.83 & 164.58 & 122.20 & 151.31 \\
\hline 1000 & 97.42 & 190.62 & 175.86 & 133.80 & 151.09 & 122.64 \\
\hline 10,000 & 97.58 & 190.61 & 228.91 & 106.26 & 189.66 & 110.00 \\
\hline
\end{tabular}

of Wu's algorithm is about $31 \mathrm{~s}$. It seems that the proposed algorithm has obvious advantages, less timeconsuming, and can quickly complete the regular superresolution restoration of single medical images.

\section{Discussion}

This paper focuses on restoration algorithm of single medical image. The following conclusions are obtained from the proposed experiment. The MSE and PSNR of proposed method are significantly improved by comparing with Liang's algorithm. Though the MSE and PSNR are slightly improved by comparing with Wu's algorithm, the restored image has an obvious improvement on burr phenomenon of local edge. ISNR is enhanced when primitive image is fused by the proposed algorithm. When the size of medical images increases, MSE and PSNR values of the proposed algorithm are the most gradual, which means that the proposed algorithm has strongest stability.

The reason that the proposed algorithm has achieved such a good restoration effect is mainly because it improves the basic idea of image fusion. In view of the fact that different restoration algorithms have different advantages for different features of an image (texture information, boundary information, smooth regions, sharp regions, etc.), multiple images are merged into one image. It allows one image to be restored more completely and accurately by using complementarity of fuzzy similarity fusion.

Research of medical image restoration algorithm is still in its infancy. It mainly focuses on the case where degradation model is linear with no noise. Systematic analysis methods and filter design methods have not yet been formed. In addition, basic theory and applied research are far from mature. Further, research direction of the algorithm is roughly divided into the following aspects.

(1) Research on improvement of algorithm

It is known from the above discussion that there are still many shortcomings in various existing algorithms, and it is necessary to further improve the algorithm. For example, how to choose the initial conditions to ensure 


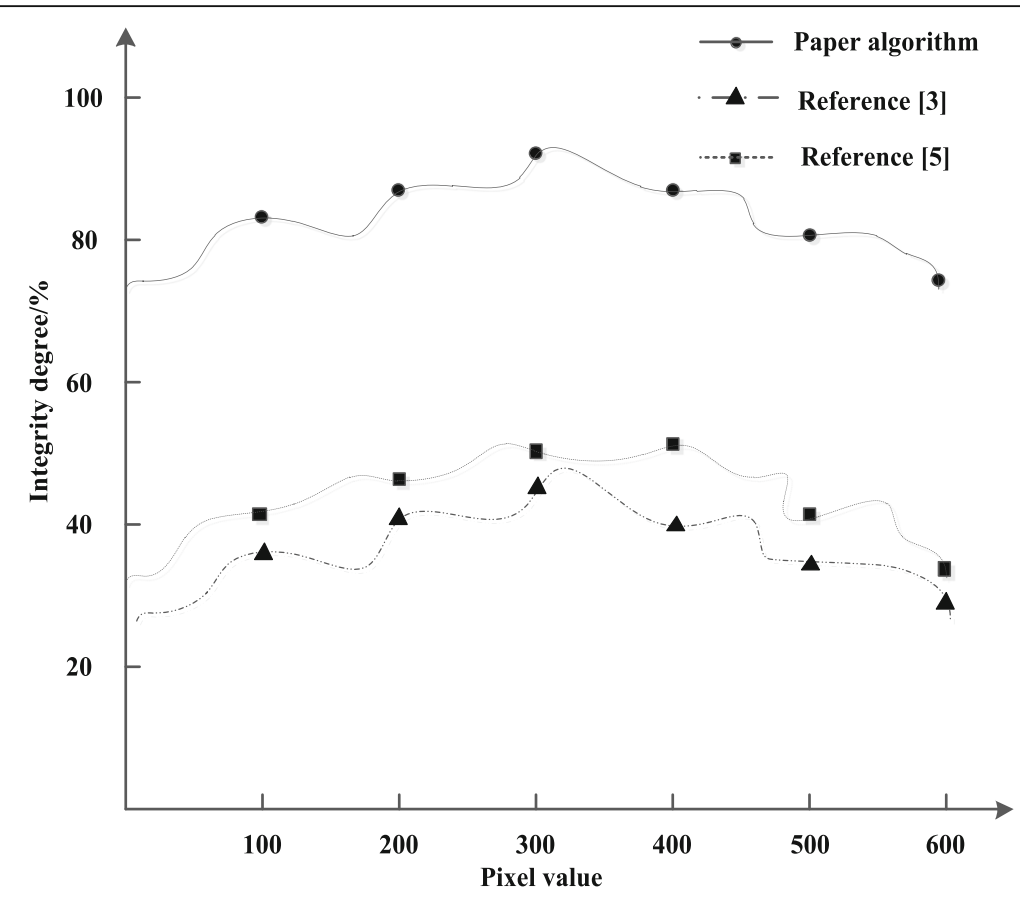

Fig. 2 Integrity comparison of image restoration

convergence of the algorithm. How to choose an algorithm termination condition to guarantee the restoration quality? How to choose the noise parameters in filter to reduce influence of noise is a hot topic in the future.

(2) Research on denoising processing algorithm
Presence of additive noise makes image restoration a challenge. Since general assumption is that only statistical properties of the noise are known, it is impossible to completely remove the noise from a degraded image. In addition, due to the presence of noise, restoration effect is not ideal. Research on image restoration algorithm

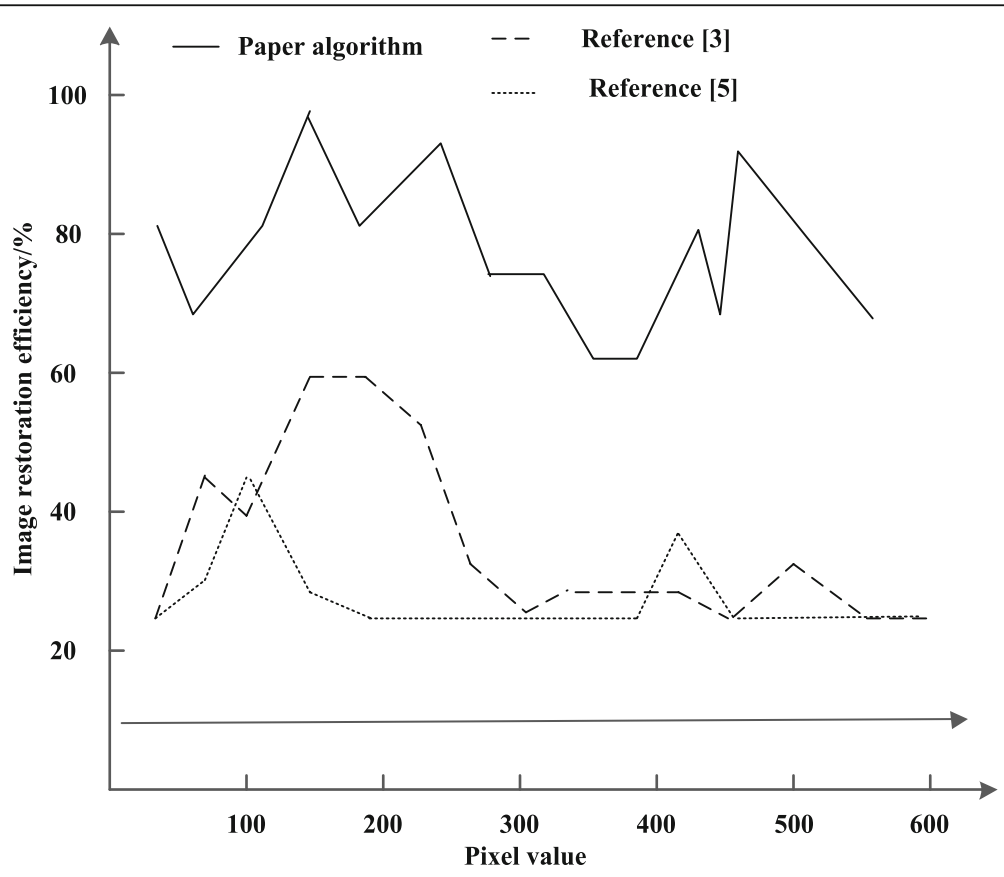

Fig. 3 Comparative analysis of image restoration efficiency 
Table 7 Image recovery time-consuming comparison

\begin{tabular}{llll}
\hline $\begin{array}{l}\text { Number of } \\
\text { experiments }\end{array}$ & Paper algorithm/s & Reference [3]/s & Reference [5]/s \\
\hline 5 & 6.2 & 23.6 & 29.6 \\
10 & 6.9 & 23.9 & 30.5 \\
15 & 8.4 & 30.9 & 28.4 \\
20 & 7.9 & 45.2 & 36.1 \\
\hline
\end{tabular}

combined with noise reduction has very practical significance. In most algorithms, noise is described as Gaussian noise, which has great limitations in practical applications. It is also an important research direction for the study of non-Gaussian cases using noise-based high-order statistical characteristics of denoising algorithms.

(3) Real-time processing algorithm

Complexity of the algorithm is an important direction that restricts its application. In related data, a method of regularized discrete periodic Radon transform is proposed to transform two-dimensional convolution into one-dimensional processing to improve algorithm' speed. And real-time processing algorithms using neural networks. Real-time nature of the algorithm is a prerequisite for its practical application.

\section{(4) Applied research}

Application of algorithms is a driving force behind research. Although image restoration algorithms have gained great applications in astronomy, medical science, and remote sensing. However, there is still a lot of work to be done to apply algorithms to general industrial image real-time detection, machine vision, and image transmission in network environment to recover criminal investigation.

\section{Conclusion}

In most electronic imaging systems, people are eager to obtain images with high resolution because these images provide more details, especially in medical research field. However, during the imaging process, there are many factors which cause descending and degradation of image quality. Such as optical system aberrations, air disturbances, motion, defocusing, discrete sampling, and system noise, which cause image blur and distortion. In the actually solution to the problem of image quality descending and degradation, image processing with superresolution is emerged. It provides many different methods to solve the above problems, and it has very important application value and broad application prospects in many fields such as medicine.
In this paper, a regularized super-resolution restoration algorithm for single medical image is proposed based on fuzzy similarity fusion. The fuzzy similarity fusion algorithm is used to obtain the fused image, and regularized super-resolution restoration is performed for the fused image, which greatly improves image restoration effect.

In addition, we will focus on the improvement of algorithm, including the strategy of convergent initial parameter choice, as well as algorithm termination condition choice.

\begin{abstract}
Abbreviations
CT: Computed tomography; DEl: Diffuse weighted imaging; HR: Highresolution; ISNR: Improved signal-to-noise ratio; LR: Low-resolution; LSI: Linear spatial invariant; MRI: Magnetic resonance imaging; MSE: Mean square error; PET: Positron emission tomography; PSF: Point spread function; PSNR: Peak signal-to-noise ratio; SPECT: Single photon emission computed tomography; SR: Super-Resolution
\end{abstract}

\section{Acknowledgements \\ We want to thank Dr. Ma from Surrey University for his help of English improvement.}

\section{Authors' contributions}

$\mathrm{XL}$ designs and codes total algorithm. WF find the motivation and analyze the results. Both authors read and approved the final manuscript.

\section{Funding}

This research is supported by following grants:

Natural Science Foundation of Inner Mongolia [No. 2018MS6010];

Foundation Science Research Start-up Fund of Inner Mongolia Agriculture University. [JC2016005];

Scientific Research Foundation for Doctors of Inner Mongolia Agriculture University. [NDYB2016-11]

\section{Availability of data and materials}

Data sharing not applicable to this article as no datasets were generated or analyzed during the current study.

\section{Competing interests}

The authors declare that they have no competing interests.

\section{Author details}

${ }^{1}$ School of Culture Management, Wuhan University of Communication, Wuhan 430000, China. ${ }^{2}$ College of Compute and Information Enginnering, Inner Mongolia Agricultrual University, Hohhot 010018, China. ${ }^{3}$ College of Information Science and Engineering, Hunan Normal University, Changsha 410081, China.

Received: 3 April 2019 Accepted: 3 October 2019

Published online: 01 November 2019

\section{References}

1. Y. Zhang, H. Wang, H. Liu, Medical image segmentation fusion based on finite naive Bayes mixture model. J. Med. Imaging Health Inform. 6(8), 18651871 (2016)

2. B. Moon, S. Yu, S. Ko, Continuous digital zooming using local self-similaritybased super-resolution for an asymmetric dual camera system. J. Opt. Soc. Am. A Opt. Image Sci. Vis. 34(6), 991-1003 (2017)

3. G. Liang, P. Shivakumara, T. Lu, Multi-spectral fusion based approach for arbitrarily oriented scene text detection in video images. IEEE Trans. Image Process. 24(11), 4488-4501 (2015)

4. X. Luo, Z. Zhang, X. Wu, A novel algorithm of remote sensing image fusion based on shift-invariant Shearlet transform and regional selection. Int. J. Electron. Commun. 70(2), 186-197 (2016)

5. D. Wu, G. Zhang, J. Lu, A fuzzy preference tree-based recommender system for personalized business-to-business e-services. IEEE Trans. Fuzzy Syst. 23(1), 29-43 (2015) 
6. S. Jeong, I. Yoon, J. Paik, Multi-frame example-based super-resolution using locally directional self-similarity. IEEE Trans. Consum. Electron. 61(3), 353-358 (2015)

7. F. Alqadah, C. Reddy, J. Hu, Biclustering neighborhood-based collaborative filtering method for top-n recommender systems. Knowl. Inf. Syst. 44(2), 475-491 (2015)

8. S. Ma, Y. Tan, G. Xu, Image completion based on fusion of patch associated matching and low-rank matrix super resolution. J. Comput. Aided Des. Comput. Graph. 27(2), 271-278 (2015)

9. M. Nilashi, O. Ibrahim, N. Ithnin, A multi-criteria recommendation system using dimensionality reduction and Neuro-fuzzy techniques. Soft. Comput. 19(11), 3173-3207 (2015)

10. S. Huang, J. Ma, P. Cheng, A hybrid multigroup coclustering recommendation framework based on information fusion. ACM Trans. Intell. Syst. Technol. 6(2), 1-22 (2015)

11. D. D'Addona, A. Ullah, D. Matarazzo, Tool-wear prediction and patternrecognition using artificial neural network and DNA-based computing. J. Intell. Manuf. 28(6), 1-17 (2017)

12. F. Xiao, An improved method for combining conflicting evidences based on the similarity measure and belief function entropy. Int. J. Fuzzy Syst. 20(1), 1-11 (2017)

13. T. Gonzálezarteaga, J. Alcantud, R. Calle, A cardinal dissensus measure based on the Mahalanobis distance. Eur. J. Oper. Res. 251(2), 575-585 (2016)

14. W. Li, X. Hu, J. Du, Adaptive remote-sensing image fusion based on dynamic gradient sparse and average gradient difference. Int. J. Remote Sens. 38(23), 7316-7332 (2017)

15. V. Kek, S. Vinodh, P. Brajesh, Rapid prototyping process selection using multi criteria decision making considering environmental criteria and its decision support system. Rapid Prototyp. J. 22(2), 225-250 (2016)

16. Y. Hang, L. Jiao, L. Fang, CRIM-FCHO: SAR image two-stage segmentation with multifeature ensemble. IEEE Trans. Geoscience Remote Sensing 54(4), 2400-2423 (2016)

17. S. Liu, W. Bai, N. Zeng, A fast fractal based compression for MRI images. IEEE Access 7, 62412-62420 (2019)

18. S. Anitha, T. Subhashini, M. Kamaraju, A novel multimodal medical image fusion approach based on phase congruency and directive contrast in NSCT domain. Int. J. Comput. Appl. 129(10), 30-35 (2015)

19. S. Liu, W. Bai, G. Liu, Parallel fractal compression method for big video data. Complexity 2018, 2016976 (2018)

20. S. Liu, Z. Pan, X. Cheng, A novel fast fractal image compression method based on distance clustering in high dimensional sphere surface. Fractals 25(4), 1740004 (2017)

21. Y. Shen, J. Wu, D. Wu, Fault diagnosis technology for three-level inverter based on reconstructive phase space and SVM. J. Power Supply 15(6), 108115 (2017)

22. L. Mengye, L. Shuai, Nucleosome positioning based on generalized relative entropy, soft computing, (online published). Soft Computing 23(19), 91759188 (2019). https://doi.org/10.1007/s00500-018-3602-2

23. R. Chou, H. Cheng, W. Sun, An improved BRISK image registration method based on adaptive threshold. Automation Instrum. 5, 172-173 (2015)

24. L. Shuai, L. Gaocheng, Z. Huiyu, A robust parallel object tracking method for illumination variations. Mobile Netw. Appl. 24(1), 5-17 (2019)

25. C. Guo, B. Wu, Z. Qin, Y. Wang, Total bounded variation fourth-order PDE image restoration model. Comput. Simul. 32(7), 239-243 (2015)

\section{Publisher's Note}

Springer Nature remains neutral with regard to jurisdictional claims in published maps and institutional affiliations.

\section{Submit your manuscript to a SpringerOpen ${ }^{\circ}$ journal and benefit from:}

- Convenient online submission

- Rigorous peer review

- Open access: articles freely available online

High visibility within the field

- Retaining the copyright to your article

Submit your next manuscript at $\boldsymbol{\nabla}$ springeropen.com 